Теорія Ймовір. та Матем. Статист. Вип. 77, 2007
Theor. Probability and Math. Statist.

No. 77, 2008, Pages 71-90 S 0094-9000(09)00748-0

Article electronically published on January 16, 2009

\title{
REPRESENTATIONS AND PROPERTIES OF WEIGHT FUNCTIONS IN TAUBERIAN THEOREMS
}

UDC 519.21

\author{
B. M. KLYKAVKA
}

\begin{abstract}
We continue the studies of weight functions in Tauberian theorems for random fields. We obtain the rate of convergence of function series in the representation of a weight function and prove a recurrence relation for weight functions in spaces of various dimensions.
\end{abstract}

\section{INTRODUCTION}

Abelian and Tauberian theorems are often used when studying asymptotic properties of random fields. Most of those theorems establish a relationship between the asymptotic behavior of the spectral function at infinity and the covariance function at zero.

The variance of the integrals of random fields is studied in the paper [1]. The explicit form of the weight function $g_{n, r, a}(|t|)$ corresponding to the spectral function

$$
\Phi_{a}(\lambda)=\Phi(a+\lambda)-\Phi(a)
$$

is obtained in [1]. A new method for the evaluation of weight functions with the help of recurrence relations is proposed in the paper 2 .

The current paper continues the studies of weight functions in Tauberian theorems for random fields. We consider the rate of convergence of function series in the representation of the function $g_{n, r, a}(|t|)$. A recurrence relation for weight functions is found for spaces of various dimensions. We present some numerical examples by using Mathematica 5.0. We also use Maple 9.5 to check all numerical results and compare them with the tables in [9] whenever possible.

\section{Some Definitions AND NOTATION}

Let $\mathbf{R}^{n}$ be the Euclidean space of dimension $n \geq 2$ and let $\xi(t), t \in \mathbf{R}^{n}$, be a realvalued measurable mean-square continuous random field, homogeneous and isotropic in the wide sense (see [4). Assume that its mean value is zero and denote the covariance function by

$$
\mathbb{B}_{n}(r)=\mathbb{B}_{n}(\|t\|)=\mathrm{E} \xi(0) \xi(t), \quad t \in \mathbf{R}^{n} .
$$

It is known that there exists a bounded nondecreasing function $\Phi(x), x \geq 0$, called the spectral function of the field $\xi(t)$, such that

$$
\mathbb{B}_{n}(r)=2^{(n-2) / 2} \Gamma\left(\frac{n}{2}\right) \int_{0}^{\infty} \frac{J_{(n-2) / 2}(r x)}{(r x)^{(n-2) / 2}} d \Phi(x),
$$

2000 Mathematics Subject Classification. Primary 60G60, 62E20, $40 \mathrm{E} 05$.

Key words and phrases. Tauberian theorems, random fields, covariance function, spectral function, weight function, rate of convergence. 
B. M. KLYKAVKA

where $J_{\nu}(z)$ is the Bessel function of the first kind and of order $\nu>-\frac{1}{2}$ (see, for example, [3, 4).

Some asymptotic properties of the variance of integrals of random fields

$$
b_{n}(r)=\operatorname{Var}\left[\int_{v(r)} \xi(t) d t\right]=(2 \pi)^{n} r^{2 n} \int_{0}^{\infty} \frac{J_{n / 2}^{2}(r x)}{(r x)^{n}} d \Phi(x),
$$

where $v(r)=\left\{t \in \mathbf{R}^{n}:|t| \leq r\right\}$ is a ball in $\mathbf{R}^{n}$, are studied in the papers [3, 4] in terms of both covariance and spectral functions.

The asymptotic behavior of the function $\Phi_{a}(\lambda):=\Phi(a+\lambda)-\Phi(a)$ as $\lambda \rightarrow+0$ is considered in the paper [1. The Tauberian theorem in [1] is stated in terms of the functionals

$$
\tilde{b}^{a}(r):=(2 \pi)^{n} \int_{0}^{\infty} \frac{J_{n / 2}^{2}(r x)}{(r x)^{n}} d \Phi_{a}(x) .
$$

It is also shown in [1] that there exists a real-valued function $g_{n, r, a}(|t|)$ such that

$$
\tilde{b}^{a}(r)=D\left[\int_{\mathbb{R}^{n}} g_{n, r, a}(|t|) \xi(t) d t\right] .
$$

Moreover

$$
g_{n, r, a}(|t|)=\frac{1}{|t|^{n / 2-1}} \int_{0}^{\infty} \underbrace{(\lambda+a)^{n / 2} J_{n / 2-1}(|t|(\lambda+a)) \frac{J_{n / 2}(r \lambda)}{(r \lambda)^{n / 2}}}_{G(\lambda)} d \lambda, \quad|t| \neq r .
$$

In what follows we use the same symbol $C$ for different constants whose exact values are not important and may vary in the course of a proof.

\section{Discussion of the PRoblem}

The rate of convergence of series in the representation of a weight function $f_{n, r, a}(|t|)$ in Tauberian theorems is obtained in the paper [5]. Some numerical results for the approximation of weight functions by partial sums of the representation are also given in [5. We are interested in obtaining similar and new results of this type for the function $g_{r, a}(|t|)$.

Using the asymptotic behavior of the Bessel function (see [7])

$$
J_{\nu}(z) \sim \sqrt{\frac{2}{\pi z}} \cos \left(z-\frac{\pi}{2} \nu-\frac{\pi}{4}\right), \quad z \rightarrow \infty,
$$

we derive the asymptotic behavior of $G(\lambda)$ given in (1), namely

$$
\begin{aligned}
G(\lambda) & \sim \frac{C}{\lambda} \cos \left(|t|(\lambda+a)-\frac{\pi n}{4}+\frac{\pi}{4}\right) \cos \left(r \lambda-\frac{n \pi}{4}-\frac{\pi}{4}\right) \\
& \sim \frac{C}{\lambda}\left(\cos \left((r+|t|) \lambda+|t| a-\frac{\pi n}{2}\right)-\sin ((|t|-r) \lambda+|t| a)\right), \quad \lambda \rightarrow \infty .
\end{aligned}
$$

Thus the integral in representation (11) converges conditionally.

The classical approach uses the Poisson formula $(7, \S 3.3])$

$$
J_{\nu}(z)=\frac{(z / 2)^{\nu}}{\Gamma(\nu+1 / 2) \Gamma(1 / 2)} \int_{-1}^{1}\left(1-x^{2}\right)^{\nu-1 / 2} \cos (z x) d x
$$

and is based on the following representation of the functional $g_{r, a}(|t|)$ :

$$
\begin{aligned}
g_{n, r, a}(|t|)= & \frac{1}{2^{n / 2-1} \Gamma\left(\frac{n-1}{2}\right) \sqrt{\pi} r^{n / 2}} \\
& \times \int_{0}^{\infty} \frac{(\lambda+a)^{n-1}}{\lambda^{n / 2}} J_{n / 2}(r \lambda) \int_{-1}^{1}\left(1-x^{2}\right)^{\frac{n-3}{2}} \cos (|t|(\lambda+a) x) d x d \lambda
\end{aligned}
$$


and on the change of variables formula

$$
\begin{aligned}
g_{n, r, a}(|t|)= & \frac{1}{2^{n / 2-1} \Gamma\left(\frac{n-1}{2}\right) \sqrt{\pi} r^{n / 2}} \\
& \times\left(\int_{-1}^{1}\left(1-x^{2}\right)^{\frac{n-3}{2}} \cos (|t| a x) \int_{0}^{\infty} \frac{(\lambda+a)^{n-1}}{\lambda^{n / 2}} J_{n / 2}(r \lambda) \cos (|t| \lambda x) d \lambda d x\right. \\
& \left.\quad-\int_{-1}^{1}\left(1-x^{2}\right)^{\frac{n-3}{2}} \sin (|t| a x) \int_{0}^{\infty} \frac{(\lambda+a)^{n-1}}{\lambda^{n / 2}} J_{n / 2}(r \lambda) \sin (|t| \lambda x) d \lambda d x\right) .
\end{aligned}
$$

The rest of the proof is devoted to evaluating the above integrals.

Unfortunately, one cannot use such a change of variables in the above integral (the same is true in the case of the functional $f_{r, a}(|t|)$ considered in [5]), since the integrals with respect to $\lambda$ are divergent for $n \in \mathbb{N}$ (this follows from the asymptotic formula (2)).

Another approach is used in the paper [1. This approach is based on a representation of the function $g_{r, a}(|t|)$ in the form of the sum of the following function series:

$$
\begin{aligned}
g_{n, r, a}(|t|)= & \frac{a^{n / 2} \Gamma\left(\frac{n-2}{2}\right)}{2^{n / 2}|t|^{n / 2}} \\
& \times \sum_{m=0}^{\infty}(-1)^{m}\left(\frac{n}{2}+m-1\right)\left(\begin{array}{c}
n+m-3 \\
m
\end{array}\right) J_{\frac{n-2}{2}+m}(|t| a) \\
& \times \sum_{k=0}^{n-1}\left(\begin{array}{c}
n-1 \\
k
\end{array}\right)\left(\frac{2}{a}\right)^{k} \Gamma\left(\frac{m+k+1}{2}\right) \\
& \times\left\{\begin{array}{cl}
\frac{2 F_{1}\left(\frac{m+k+1}{2}, \frac{3+k-m-n}{2} ; \frac{n}{2}+1 ;\left(\frac{r}{|t|}\right)^{2}\right)}{\left.|t|^{k} \Gamma \frac{n}{2}+1\right) \Gamma\left(\frac{m+n-k-1}{2}\right)}, & |t|>r, \\
\frac{|t|^{m+1}{ }_{2}\left(\frac{m+k+1}{2}, \frac{m+k+1-n}{2} ; \frac{n}{2}+m ;\left(\frac{|t|}{r}\right)^{2}\right)}{r^{m+k+1} \Gamma\left(\frac{n}{2}+m\right) \Gamma\left(\frac{n-m-k+1}{2}\right)}, & |t|<r,
\end{array}\right.
\end{aligned}
$$

where the corresponding term vanishes in the cases of $|t|<r$ and

$$
(m+k-n-1) / 2 \in \mathbf{N} \cup\{0\},
$$

since $\Gamma((n-m-k+1) / 2)=\infty$.

We will use the following notation:

$$
\begin{aligned}
d_{m}(n, r, a, t):= & (-1)^{m}\left(\frac{n}{2}+m-1\right)\left(\begin{array}{c}
n+m-3 \\
m
\end{array}\right) J_{\frac{n-2}{2}+m}(|t| a) \\
\times & \sum_{k=0}^{n-1}\left(\begin{array}{c}
n-1 \\
k
\end{array}\right)\left(\frac{2}{a}\right)^{k} \Gamma\left(\frac{m+k+1}{2}\right) \\
& \times \frac{|t|^{m+1}{ }_{2} F_{1}\left(\frac{m+k+1}{2}, \frac{m+k+1-n}{2} ; \frac{n}{2}+m ;\left(\frac{|t|}{r}\right)^{2}\right)}{r^{m+k+1} \Gamma\left(\frac{n}{2}+m\right) \Gamma\left(\frac{n-m-k+1}{2}\right)}, \\
s_{m}(n, r, a,|t|):= & (-1)^{m}\left(\frac{n}{2}+m-1\right)\left(\begin{array}{c}
n+m-3 \\
m
\end{array}\right) J_{\frac{n-2}{2}+m}(|t| a) \\
& \times \sum_{k=0}^{n-1}\left(\begin{array}{c}
n-1 \\
k
\end{array}\right)\left(\frac{2}{a}\right)^{k} \Gamma\left(\frac{m+k+1}{2}\right) \\
& \times \frac{{ }_{2} F_{1}\left(\frac{m+k+1}{2}, \frac{3+k-m-n}{2} ; \frac{n}{2}+1 ;\left(\frac{r}{|t|}\right)^{2}\right)}{|t|^{k} \Gamma\left(\frac{n}{2}+1\right) \Gamma\left(\frac{m+n-k-1}{2}\right)} .
\end{aligned}
$$




\section{ASYMPtotic PROPERTIES OF A HYPERGEOMETRIC FUNCTION}

We need some properties of the hypergeometric function

$$
{ }_{2} F_{1}(a, b, c ; z)
$$

to evaluate the sum in (44) (see [8]). First, consider the asymptotic behavior of

$$
{ }_{2} F_{1}\left(\frac{m+k+1}{2}, \frac{m+k+1-n}{2}, \frac{n}{2}+m ;\left(\frac{|t|}{r}\right)^{2}\right)
$$

as $m \rightarrow \infty$. We use the Stirling formula,

$$
\Gamma(k+1) \sim \sqrt{2 \pi} k^{k+1 / 2} e^{-k} e^{\theta_{k} / k} \quad \text { and } \quad k ! \sim \sqrt{2 \pi} k^{k+1 / 2} e^{-k} e^{\theta_{k} / k},
$$

where $\theta_{k} \in\left(0 ; \frac{1}{12}\right)$.

The Watson result (see [8, §2.3.2]) is often helpful when deriving the asymptotic results. Following Watson's method with

$$
a=\frac{k+1}{2}, \quad c=\frac{n}{2}+1, \quad b=\frac{k-n+3}{2}, \quad \lambda=\frac{m}{2}, \quad \frac{2}{1-z}=\left(\frac{|t|}{r}\right)^{2},
$$

we obtain

$$
\begin{aligned}
{ }_{2} F_{1} & \left(\frac{m+k+1}{2}, \frac{m+k+1-n}{2}, \frac{n}{2}+m ;\left(\frac{|t|}{r}\right)^{2}\right) \\
& \sim \frac{\sqrt{2 \pi} \Gamma(n / 2+m) 2^{k+2-n / 2}}{\Gamma\left(\frac{k-n+1+m}{2}\right) \Gamma\left(n+\frac{m-k-1}{2}\right) m^{1 / 2}}\left(\frac{r}{|t|}\right)^{m+k+1} O\left(C^{m}\right) \\
& \sim O\left(\frac{m^{n / 2+m-1 / 2} C^{m}}{m^{1 / 2} m^{(m+k-n) / 2} m^{(m-k+2 n-2) / 2}}\right)=O\left(C^{m}\right)
\end{aligned}
$$

as $m \rightarrow \infty$. This formula holds in the domain $z \in \mathbf{C} \backslash(-\infty, 1)$ and, unfortunately, cannot be used directly in our case. Nevertheless the asymptotic behavior of order $O\left(C^{m}\right)$ can be proved.

Lemma 4.1. For $|t|<r$,

$$
{ }_{2} F_{1}\left(\frac{m+k+1}{2}, \frac{m+k+1-n}{2}, \frac{n}{2}+m ;\left(\frac{|t|}{r}\right)^{2}\right)=O\left(C^{m}\right), \quad m \rightarrow \infty .
$$

Proof. Using the definition of the hypergeometric function (see [5, 3]),

$$
\begin{aligned}
{ }_{2} F_{1} & \left(\frac{m+k+1}{2}, \frac{m+k+1-n}{2}, \frac{n}{2}+m ;\left(\frac{|t|}{r}\right)^{2}\right)=\sum_{l=0}^{\infty} \frac{\left(\frac{m+k+1}{2}\right)_{l}\left(\frac{m+k+1-n}{2}\right)_{l}}{\left(\frac{n}{2}+m\right)_{l} l !} z^{l} \\
& =\sum_{l=0}^{\infty} \frac{\Gamma\left(\frac{m+k+1}{2}+l\right) \Gamma\left(\frac{n}{2}+m\right) \Gamma\left(\frac{m+k+1-n}{2}+l\right) z^{l}}{\Gamma\left(\frac{m+k+1}{2}\right) \Gamma\left(\frac{n}{2}+m+l\right) \Gamma\left(\frac{m+k+1-n}{2}\right) \Gamma(l+1)} \\
& =1+\frac{\Gamma\left(\frac{n}{2}+m\right)}{\Gamma\left(\frac{m+k+1}{2}\right) \Gamma\left(\frac{m+k+1-n}{2}\right)} \sum_{l=1}^{\infty} \frac{\Gamma\left(\frac{m+k+1}{2}+l\right) \Gamma\left(\frac{m+k+1-n}{2}+l\right) z^{l}}{\Gamma\left(\frac{n}{2}+m+l\right) \Gamma(l+1)}
\end{aligned}
$$

According to Stirling's formula, we get

$$
\frac{\Gamma\left(\frac{n}{2}+m\right)}{\Gamma\left(\frac{m+k+1}{2}\right) \Gamma\left(\frac{m+k+1-n}{2}\right)} \sim \frac{2^{m+k-n / 2} m^{n-k-1 / 2}}{\sqrt{2 \pi}}
$$


as $m \rightarrow \infty$. Using Stirling's formula again, we rearrange and estimate the series:

$$
\begin{aligned}
\sum_{l=1}^{\infty} & \frac{\Gamma\left(\frac{m+k+1}{2}+l\right) \Gamma\left(\frac{m+k+1-n}{2}+l\right) z^{l}}{\Gamma\left(\frac{n}{2}+m+l\right) \Gamma(l+1)} \\
& \leq C \sum_{l=1}^{\infty} \frac{\left(\frac{m+k-1}{2}+l\right)^{1 / 2}\left(\frac{m+k-n-1}{2}+l\right)^{(m+k-n) / 2} b^{(m+k-n-1) / 2} z^{l}}{l^{1 / 2}\left(\frac{n}{2}+m+l-1\right)^{(m+n-k) / 2} a^{(n+m-k-1) / 2}} \\
& \leq C \sum_{l=1}^{\infty} \frac{C_{1}^{m} z^{l}}{l^{1 / 2} m^{n-k-1 / 2}} .
\end{aligned}
$$

After some algebra in (7) and (8) we complete the proof of the lemma.

Theorem 4.1. For $|t|<r$,

$$
\begin{gathered}
\sum_{m=N}^{\infty} d_{m}(n, r, a, t)=O\left(\sum_{m=N}^{\infty} \frac{C^{m}}{m^{m-n / 2+2}}\right), \quad N \rightarrow \infty, \\
\sum_{m=N}^{\infty} d_{m}(n, r, a, t)=O\left(\frac{C^{N}}{N^{N(1-\varepsilon)}}\right), \quad N \rightarrow \infty,
\end{gathered}
$$

where $\varepsilon>0$ is arbitrary.

Proof. Consider the asymptotic behavior of the factors in representation (4) for the function $g_{n, r, a}(|t|)$ :

$$
\left(\begin{array}{c}
n+m-3 \\
m
\end{array}\right) \sim \frac{m^{n-3}}{(n-3) !} .
$$

Applying formula [5, (22)] we obtain

$$
J_{n / 2+m-1}(|t| a)=O\left(\frac{C^{m}}{m^{n / 2+m-1 / 2}}\right), \quad m \rightarrow \infty .
$$

Consider

$$
\begin{aligned}
& d_{k}^{*}(m, n, r, a, t) \\
& \quad:=\sum_{k=0}^{n-1}\left(\begin{array}{c}
n-1 \\
k
\end{array}\right)\left(\frac{2}{a}\right)^{k} \Gamma\left(\frac{m+k+1}{2}\right) \frac{|t|^{m+1}{ }_{2} F_{1}\left(\frac{m+k+1}{2}, \frac{m+k+1-n}{2} ; \frac{n}{2}+m ;\left(\frac{|t|}{r}\right)^{2}\right)}{r^{m+k+1} \Gamma\left(\frac{n}{2}+m\right) \Gamma\left(\frac{n-m-k+1}{2}\right)} .
\end{aligned}
$$

We use Stirling's formula and follow the lines of the above proof:

$$
d_{k}^{*}(m, n, r, a, t)=\sum_{k=0}^{n-1} O\left(\frac{C^{m}}{m^{n-k-1 / 2}}\right)=O\left(\frac{C^{m}}{m^{1 / 2}}\right), \quad m \rightarrow \infty .
$$

The asymptotic formula (10) follows from

$$
\begin{aligned}
\sum_{m=N}^{\infty} \frac{C^{m}}{m^{(m-n / 2+2)}} & \leq \sum_{m=N}^{\infty} \frac{C^{m}}{m^{(m-\varepsilon m)}} \leq \sum_{m=N}^{\infty}\left(\frac{C}{N^{(1-\varepsilon)}}\right)^{m}=\frac{C^{N} N^{1-\varepsilon}}{N^{N(1-\varepsilon)}\left(N^{1-\varepsilon}-C\right)} \\
& =O\left(\frac{C^{N}}{N^{N(1-\varepsilon)}}\right), \quad N \rightarrow \infty,
\end{aligned}
$$

for all $\varepsilon>0$.

Now we study the asymptotic behavior of

$$
{ }_{2} F_{1}\left(\frac{m+k+1}{2}, \frac{3+k-m-n}{2} ; \frac{n}{2}+1 ;\left(\frac{r}{|t|}\right)^{2}\right) \quad \text { as } m \rightarrow \infty .
$$


We apply the result of $[8, \S 2.3 .2]$ again. Similarly to the above-considered case we get

$$
{ }_{2} F_{1}\left(\frac{m+k+1}{2}, \frac{3+k-m-n}{2} ; \frac{n}{2}+1 ;\left(\frac{r}{|t|}\right)^{2}\right) \sim O\left(\frac{C^{m}}{m^{(n+1) / 2}}\right), \quad m \rightarrow \infty .
$$

This result holds in the domain $z \in \mathbf{C} \backslash(-\infty, 1)$ and cannot be used directly in our case. Nevertheless we prove that the asymptotic behavior is of order $O\left(C^{m} / m^{(n+1) / 2}\right)$.

Lemma 4.2. For $|t|>r$,

$$
\begin{gathered}
{ }_{2} F_{1}\left(\frac{m+k+1}{2}, \frac{3+k-m-n}{2}, \frac{n}{2}+1 ;\left(\frac{r}{|t|}\right)^{2}\right)=O\left(\frac{C^{m}}{m^{n / 2+1 / 2}}\right), \\
m \rightarrow \infty .
\end{gathered}
$$

Proof. First we consider the case where $(m+n-k-3)$ is an even number. Let

$$
m+n-k-3=2 p .
$$

Then

$$
\begin{aligned}
\left|{ }_{2} F_{1}\left(\frac{m+k+1}{2},-p, \frac{n}{2}+1 ; z\right)\right| & =\left|\sum_{l=0}^{p} \frac{\Gamma\left(\frac{m+k+1}{2}+l\right)(-1)^{l} p ! \Gamma\left(\frac{n}{2}+1\right) z^{l}}{\Gamma\left(\frac{m+k+1}{2}\right) \Gamma\left(\frac{n}{2}+1+l\right)(p-l) ! l !}\right| \\
& \leq \frac{\Gamma\left(\frac{n}{2}+1\right)}{\Gamma\left(\frac{m+k+1}{2}\right)} \sum_{l=0}^{p}\left(\begin{array}{c}
p \\
l
\end{array}\right) z^{l} \frac{\Gamma\left(\frac{m+k+1}{2}+l\right)}{\Gamma\left(\frac{n}{2}+1+l\right)} \\
& \leq \frac{\Gamma\left(\frac{m+k+1}{2}+p\right) \Gamma\left(\frac{n}{2}+1\right)}{\Gamma\left(\frac{n}{2}+1+p\right) \Gamma\left(\frac{m+k+1}{2}\right)}(1+z)^{p},
\end{aligned}
$$

since

$$
\frac{\Gamma\left(\frac{m+k+1}{2}+l\right)}{\Gamma\left(\frac{n}{2}+1+l\right)} \leq \frac{\Gamma\left(\frac{m+k+1}{2}+p\right)}{\Gamma\left(\frac{n}{2}+1+p\right)}
$$

for all $l=0, \ldots, p$. By Stirling's formula,

$$
\begin{gathered}
\frac{\Gamma\left(\frac{m+k+1}{2}+p\right)}{\Gamma\left(\frac{n}{2}+1+p\right)}=\frac{\Gamma\left(m-2+\frac{n}{2}+1\right)}{\Gamma\left(n+\frac{m-k-3}{2}+1\right)} \sim C^{m} \frac{m^{m+n / 2-3 / 2}}{m^{m / 2+n-k / 2-1}}=C^{m} m^{(m-n+k-1) / 2}, \\
{ }_{2} F_{1}\left(\frac{m+k+1}{2}+l,-p, \frac{n}{2}+1 ; z\right)=O\left(\frac{C^{m}}{m^{(n+1) / 2}}\right) .
\end{gathered}
$$

Now let $(m+n-k-3)$ be an odd number. Then

$$
\begin{aligned}
{ }_{2} F_{1}( & \left.\frac{m+k+1}{2}, \frac{3+k-m-n}{2}, \frac{n}{2}+1 ; z\right) \\
= & \sum_{l=0}^{\left[\frac{m+n-k-3}{2}\right]+1} \frac{\left(\frac{m+k+1}{2}\right)_{l}\left(\frac{3+k-m-n}{2}\right)_{l}}{\left(\frac{n}{2}+1\right)_{l} l !} z^{l} \\
& +\sum_{\left[\frac{m+n-k-3}{2}\right]+2}^{\infty} \frac{\left(\frac{m+k+1}{2}\right)_{l}\left(\frac{3+k-m-n}{2}\right)_{l}}{\left(\frac{n}{2}+1\right)_{l} l !} z^{l} .
\end{aligned}
$$


We have

$$
\begin{aligned}
& \left|\left(\frac{m+k+1}{2}\right)_{l}\left(\frac{3+k-m-n}{2}\right)_{l}\right| \\
& =\mid\left(\frac{m+k+1}{2}\right) \ldots\left(\frac{m+k+1}{2}+l-1\right) \\
& \quad \times\left(\frac{m+n-k-3}{2}\right) \ldots\left(\frac{m+n-k-3}{2}-l+1\right) \mid \\
& \leq \mid\left(\frac{m+k+1}{2}+\frac{1}{2}\right) \ldots\left(\frac{m+k+1}{2}+l-1+\frac{1}{2}\right) \\
& \quad \times \quad\left|\left(\frac{m+n-k-3}{2}+\frac{1}{2}\right) \ldots\left(\frac{m+n-k-3}{2}-l+1+\frac{1}{2}\right)\right|
\end{aligned}
$$

Thus

$$
\begin{aligned}
& \left|\sum_{l=0}^{\left[\frac{m+n-k-3}{2}\right]+1} \frac{\left(\frac{m+k+1}{2}\right)_{l}\left(\frac{3+k-m-n}{2}\right)_{l}}{\left(\frac{n}{2}+1\right)_{l} l !} z^{l}\right| \leq \sum_{l=0}^{\frac{m+n-k-2}{2}} \frac{\left(\frac{m+k+2}{2}\right)_{l}\left(\frac{2+k-m-n}{2}\right)_{l}}{\left(\frac{n}{2}+1\right)_{l} l !} z^{l} \\
& =O\left(\frac{C^{m}}{m^{(n+1) / 2}}\right), \quad m \rightarrow \infty \text {. }
\end{aligned}
$$

Now we estimate the second term in (14):

$$
\begin{aligned}
& \sum_{l=\left[\frac{m+n-k-3}{2}\right]+2}^{\infty} \frac{\left(\frac{m+k+1}{2}\right)_{l}\left(\frac{3+k-m-n}{2}\right)_{l}}{\left(\frac{n}{2}+1\right)_{l} l !} z^{l} \\
= & \sum_{l=\left[\frac{m+n-k-3}{2}\right]+2}^{\infty} \frac{\Gamma\left(\frac{m+k+1}{2}+l\right) \Gamma\left(\frac{n}{2}+1\right)\left(-\frac{m+n-k-3}{2}\right)\left(-\frac{m+n-k-3}{2}+1\right) \ldots}{\Gamma\left(\frac{m+k+1}{2}\right) \Gamma\left(\frac{n}{2}+1+l\right) \Gamma(l+1)} \\
= & \times\left(-\frac{1}{2}\right)\left(\frac{1}{2}\right) \ldots\left(-\frac{m+n-k-3}{2}+l-1\right) z^{l} \\
= & \frac{(-1)^{\frac{m+n-k-2}{2}} \Gamma\left(\frac{m+n-k-1}{2}\right) \Gamma\left(\frac{n}{2}+1\right) z^{\frac{m+n-k-2}{2}}}{\pi \Gamma\left(\frac{m+k+1}{2}\right)} \\
& \times \sum_{s=1}^{\infty} \frac{\Gamma\left(s+m+\frac{m-1}{2}\right) \Gamma\left(s+\frac{1}{2}\right)}{\Gamma\left(s+n+\frac{m-k}{2}\right) \Gamma\left(s+\frac{m+n-k}{2}\right)}
\end{aligned}
$$

and

$$
\begin{aligned}
\sum_{s=1}^{\infty} \frac{\Gamma\left(s+m+\frac{n-1}{2}\right) \Gamma\left(s+\frac{1}{2}\right)}{\Gamma\left(s+n+\frac{m-k}{2}\right) \Gamma\left(s+\frac{m+n-k}{2}\right)} & \leq C^{m} \sum_{s=1}^{\infty} \frac{\frac{b^{\frac{m-n+k-1}{2+\frac{m-n-2}{2}}}\left(s+m+\frac{n}{2}-1\right)}{2} z^{s}}{\left(s+\frac{m+n-k-2}{2}\right)^{n-k}} \\
& \leq \frac{C^{m}}{\left(\frac{m+n-k-2}{2}\right)^{n-k}} \sum_{s=1}^{\infty} z^{s} .
\end{aligned}
$$


Representation (16) and Stirling's formula imply that

$$
\begin{aligned}
& \left|\sum_{l=\left[\frac{m+n-k-3}{2}\right]+2}^{\infty} \frac{\left(\frac{m+k+1}{2}\right)_{l}\left(\frac{3+k-m-n}{2}\right)_{l}}{\left(\frac{n}{2}+1\right)_{l} l !} z^{l}\right| \\
& \quad=O\left(\frac{C^{m} \Gamma\left(\frac{m+n-k-1}{2}\right)}{\left(\frac{m+n-k-2}{2}\right)^{n-k} \Gamma\left(\frac{m+k+1}{2}\right)}\right)=O\left(\frac{C^{m}}{m^{(n+1) / 2}}\right), \quad m \rightarrow \infty .
\end{aligned}
$$

Substituting (15) and (17) in (14) we complete the proof of the lemma.

Theorem 4.2. If $|t|>r$, then

$$
\begin{gathered}
\sum_{m=N}^{\infty} s_{m}(n, r, a, t)=O\left(\sum_{m=N}^{\infty} \frac{C^{m}}{m^{m-n / 2+2}}\right), \quad N \rightarrow \infty \\
\sum_{m=N}^{\infty} s_{m}(n, r, a, t)=O\left(\frac{C^{N}}{N^{N(1-\varepsilon)}}\right), \quad N \rightarrow \infty
\end{gathered}
$$

for all $\varepsilon>0$.

Proof. First we consider the asymptotic behavior of the expression for $s_{m}(n, r, a, t)$ in formula (하). Put

$s_{m}^{\star}(n, r, a, t)=\sum_{k=0}^{n-1}\left(\begin{array}{c}n-1 \\ k\end{array}\right)\left(\frac{2}{a}\right)^{k} \Gamma\left(\frac{m+k+1}{2}\right) \frac{{ }_{2} F_{1}\left(\frac{m+k+1}{2}, \frac{3+k-m-n}{2} ; \frac{n}{2}+1 ;\left(\frac{r}{|t|}\right)^{2}\right)}{|t|^{k} \Gamma\left(\frac{n}{2}+1\right) \Gamma\left(\frac{m+n-k-1}{2}\right)}$.

Using Stirling's formula and Lemma 4.2 we obtain

$$
s_{m}^{\star}(n, r, a, t)=\sum_{k=0}^{n-1} O\left(\frac{C^{m}}{m^{n-k-1 / 2}}\right)=O\left(\frac{C^{m}}{m^{1 / 2}}\right) .
$$

Applying bounds (11), (12), and (20) we prove relation (18).

Equality (19) is proved similarly to the proof of Theorem 4.1.

\section{ReCuRREnCE RELAtions FOR The Weight FunCtions $g_{n, r, a}(t)$}

Recalling the well-known identities for the derivatives of the Bessel functions (see $§ 3.2$ in [7), we get

$$
\frac{d}{d z}\left(z^{\nu} J_{\nu}(z)\right)=z^{\nu} J_{\nu-1}(z), \quad \frac{d}{d z}\left(\frac{J_{\nu}(z)}{z^{\nu}}\right)=-\frac{J_{\nu+1}(z)}{z^{\nu}}
$$

Integrating by parts the integral in representation (9) (see [1]) for the weight function $g_{n, r, a}(t)$ and considering the asymptotic behavior of the Bessel function at the origin and 
at infinity, we get

$$
\begin{aligned}
g_{n, r, a}(|t|)= & \frac{1}{|t|^{n / 2-1}} \int_{0}^{\infty}(\lambda+a)^{n / 2} J_{n / 2-1}(|t|(\lambda+a)) \frac{J_{n / 2}(r \lambda)}{(r \lambda)^{n / 2}} d \lambda \\
= & \left.\frac{J_{n / 2}(r \lambda)}{|t|^{n}(r \lambda)^{n / 2}}(|t|(\lambda+a))^{n / 2} J_{n / 2}(|t|(\lambda+a))\right|_{0} ^{\infty} \\
& +\frac{r}{|t|^{n}} \int_{0}^{\infty}(|t|(\lambda+a))^{n / 2} J_{n / 2}(|t|(\lambda+a)) \frac{J_{n / 2+1}(r \lambda)}{(r \lambda)^{n / 2}} d \lambda \\
= & -\left(\frac{a}{2|t|}\right)^{n / 2} \cdot \frac{J_{n / 2}(|t| a)}{\Gamma\left(\frac{n}{2}+1\right)} \\
& +\frac{r}{|t|^{n}} \int_{0}^{\infty}(|t|(\lambda+a))^{n / 2} J_{n / 2}(|t|(\lambda+a)) \frac{J_{n / 2+1}(r \lambda)}{(r \lambda)^{n / 2}} d \lambda .
\end{aligned}
$$

Furthermore,

$$
\begin{aligned}
g_{n, r, a}(|t|)= & -\left(\frac{a}{2|t|}\right)^{n / 2} \cdot \frac{J_{n / 2}(|t| a)}{\Gamma\left(\frac{n}{2}+1\right)} \\
& +\frac{r^{2}}{|t|^{n+1}} \int_{0}^{\infty}(|t|(\lambda+a))^{n / 2+1} J_{n / 2}(|t|(\lambda+a)) \frac{J_{n / 2+1}(r \lambda)}{(r \lambda)^{n / 2+1}} d \lambda \\
& -\frac{a r^{2}}{|t|^{n}} \int_{0}^{\infty}(|t|(\lambda+a))^{n / 2} J_{n / 2}(|t|(\lambda+a)) \frac{J_{n / 2+1}(r \lambda)}{(r \lambda)^{n / 2+1}} d \lambda \\
= & -h_{n, a}(|t|)+r^{2} g_{n+2, r, a}(|t|)-r^{2} G_{n}(r, a,|t|),
\end{aligned}
$$

where

$$
\begin{gathered}
h_{n, a}(|t|)=\left(\frac{a}{2|t|}\right)^{n / 2} \cdot \frac{J_{\frac{n}{2}}(|t| a)}{\Gamma\left(\frac{n}{2}+1\right)}, \\
G_{n}(r, a,|t|)=\frac{a}{|t|^{n}} \int_{0}^{\infty}(|t|(\lambda+a))^{n / 2} J_{n / 2}(|t|(\lambda+a)) \frac{J_{n / 2+1}(r \lambda)}{(r \lambda)^{n / 2+1}} d \lambda .
\end{gathered}
$$

The recurrence relation implies the following result.

Lemma 5.1. For an arbitrary $n \geq 1$,

$$
g_{n+2, r, a}(|t|)=\frac{g_{n, r, a}(|t|)+h_{n, a}(|t|)}{r^{2}}+G_{n}(r, a,|t|),
$$

where $h_{n, a}(|t|)$ and $G_{n}(r, a,|t|)$ are defined in (21) and (22), respectively.

Corollary 5.1. Let $n>m \geq 1$. Then

(i) if $n$ and $m$ are even numbers,

$$
g_{n, r, a}(|t|)=\frac{g_{m, r, a}(|t|)}{r^{n-m}}+\sum_{k=m / 2+1}^{n / 2} \frac{G_{2 k-2}(r, a,|t|)}{r^{n-2 k}}+\sum_{k=m / 2+1}^{n / 2} \frac{h_{2 k-2, a}(|t|)}{r^{n-2 k+2}} ;
$$

(ii) if $n$ and $m$ are odd numbers,

$$
g_{n, r, a}(|t|)=\frac{g_{m, r, a}(|t|)}{r^{n-m}}+\sum_{k=(m+1) / 2}^{(n-1) / 2} \frac{G_{2 k-1}(r, a,|t|)}{r^{n-2 k-1}}+\sum_{k=(m+1) / 2}^{(n-1) / 2} \frac{h_{2 k-1, a}(|t|)}{r^{n-2 k+1}} .
$$




\section{Evaluation of $g_{1, r, a}(|t|)$}

Lemma 6.1. The function $g_{1, r, a}(|t|)$ is given by

$$
g_{1, r, a}(|t|)=\frac{\log \left(\frac{|t|-r}{|t|+r}\right) \sin (a|t|)}{\pi r}
$$

for $|t|>r$ and by

$$
g_{1, r, a}(|t|)=\frac{\pi \cos (a|t|)+\log \left(\frac{r-|t|}{r+|t|}\right) \sin (a|t|)}{\pi r}
$$

for $|t|<r$.

Proof. Applying [7, §3.4] we get

$$
J_{\frac{1}{2}}(z)=\sqrt{\frac{2}{\pi z}} \sin (z), \quad J_{-\frac{1}{2}}(z)=\sqrt{\frac{2}{\pi z}} \cos (z) .
$$

Hence

$$
g_{1, r, a}(|t|)=\int_{0}^{\infty} \frac{J_{\frac{1}{2}}(r \lambda)}{\sqrt{r \lambda}} \sqrt{|t|(\lambda+a)} J_{-\frac{1}{2}}(|t|(\lambda+a)) d \lambda=\frac{2}{\pi} \int_{0}^{\infty} \frac{\cos (|t|(a+\lambda)) \sin (r \lambda)}{r \lambda} d \lambda .
$$

Now we use Mathematica 5.0 and [9] to obtain equalities (23) and (24).

\section{Evaluation of $g_{2, r, a}(|t|)$}

Lemma 7.1. If $|t| \neq r$, then

$$
\begin{aligned}
g_{2, r, a}(|t|)= & \frac{2}{\pi r^{2}} \int_{0}^{\min (1, r /|t|)} \frac{a\left(r^{2}-|t|^{2} x^{2}\right) \cos (a|t| x)-|t| x \sin (a|t| x)}{\sqrt{1-x^{2}} \sqrt{r^{2}-|t|^{2} x^{2}}} d x \\
& +\frac{J_{0}(a|t|)-a|t| J_{1}(a|t|)}{r^{2}} \\
& +\frac{2}{\pi r^{2}} \int_{\min (1, r /|t|)}^{1} \frac{a\left(|t|^{2} x^{2}-r^{2}\right) \sin (a|t| x)-|t| x \cos (a|t| x)}{\sqrt{1-x^{2}} \sqrt{|t|^{2} x^{2}-r^{2}}} d x .
\end{aligned}
$$

Proof. We use the Poisson integral (3) to evaluate $g_{2, r, a}(|t|)$ :

$$
\begin{aligned}
g_{2, r, a}(|t|) & =\int_{0}^{\infty} \frac{\lambda+a}{r \lambda} J_{0}(|t|(\lambda+a)) J_{1}(r \lambda) d \lambda \\
& =\frac{1}{r \pi} \int_{0}^{\infty} \frac{\lambda+a}{\lambda} J_{1}(r \lambda) \int_{-1}^{1} \frac{\cos (|t|(\lambda+a) x)}{\sqrt{1-x^{2}}} d x d \lambda \\
& =\frac{1}{r \pi} \int_{-1}^{1} \frac{1}{\sqrt{1-x^{2}}} \int_{0}^{\infty} \frac{\lambda+a}{\lambda} J_{1}(r \lambda) \cos (|t|(\lambda+a) x) d \lambda d x .
\end{aligned}
$$

Now we justify the change of order of integration. The expression (27) can be rewritten as follows:

$$
\begin{aligned}
\int_{0}^{\infty} & \int_{-1}^{1} Q_{|t|, r, a}(x, \lambda) d x d \lambda \\
& =\int_{0}^{C} \int_{-1}^{1} Q_{|t|, r, a}(x, \lambda) d x d \lambda+\int_{C}^{\infty} \int_{-1}^{1} Q_{|t|, r, a}(x, \lambda) d x d \lambda
\end{aligned}
$$

where

$$
Q_{|t|, r, a}(x, \lambda):=\frac{\lambda+a}{\lambda \sqrt{1-x^{2}}} J_{1}(r \lambda) \cos (|t|(\lambda+a) x) .
$$


The domain of integration for the integral (28) is a compact set, and the integral converges absolutely. Thus the change of order is justified for this integral.

Now we show that

$$
\int_{C}^{\infty} \int_{-1}^{1} Q_{|t|, r, a}(x, \lambda) d x d \lambda \rightarrow 0, \quad \int_{-1}^{1} \int_{C}^{\infty} Q_{|t|, r, a}(x, \lambda) d \lambda d x \rightarrow 0
$$

as $C \rightarrow+\infty$. The first part of (29) follows from representation (26):

$$
\int_{C}^{\infty} \int_{-1}^{1} Q_{|t|, r, a}(x, \lambda) d x d \lambda=\pi \int_{C}^{\infty} \frac{\lambda+a}{\lambda} J_{0}(|t|(\lambda+a)) J_{1}(r \lambda) d \lambda
$$

and from the known asymptotic behavior of the Bessel function (see [5, (2)]). We introduce the notation

$$
\begin{aligned}
& \int_{C}^{\infty} \frac{\lambda+a}{\lambda} J_{1}(r \lambda) \cos (|t|(\lambda+a) x) d \lambda \\
& =\cos (|t| a x) \underbrace{\int_{C}^{\infty} \frac{\lambda+a}{\lambda} J_{1}(r \lambda) \cos (|t| \lambda x) d \lambda}_{I_{1}}-\sin (|t| a x) \underbrace{\int_{C}^{\infty} \frac{\lambda+a}{\lambda} J_{1}(r \lambda) \sin (|t| \lambda x) d \lambda .}_{I_{2}}
\end{aligned}
$$

to prove the second part of (29).

It is known that

$$
J_{\nu}(z)=\frac{H_{\nu}^{(1)}(z)+H_{\nu}^{(2)}(z)}{2}
$$

where $H_{\nu}^{(k)}(z), k=1,2$, are Bessel functions of the third kind (see [7, (1), §3.61]).

Now we use the asymptotic representations of the Bessel functions (3) and (4) for $\nu=1$ and $p=1$ (see [7, $\S 7.2])$. Then

$$
H_{1}^{(k)}(z)=\sqrt{\frac{2}{\pi z}} e^{ \pm i(z-3 \pi / 4)}\left(1+O\left(\frac{1}{z}\right)\right)
$$

for $k=1$ and 2, respectively.

Consider the following bound:

$$
\begin{aligned}
& \left|I_{1}\right| \leq\left|\int_{C}^{\infty} \frac{\lambda+a}{\lambda \sqrt{2 \pi r \lambda}} e^{i r \lambda} \cos (|t| \lambda x) d \lambda\right|+\left|\int_{C}^{\infty} \frac{\lambda+a}{\lambda \sqrt{2 \pi r \lambda}} e^{i r \lambda} \cos (|t| \lambda x) O\left(\frac{1}{\lambda}\right) d \lambda\right| \\
& +\left|\int_{C}^{\infty} \frac{\lambda+a}{\lambda \sqrt{2 \pi r \lambda}} e^{-i r \lambda} \cos (|t| \lambda x) d \lambda\right|+\left|\int_{C}^{\infty} \frac{\lambda+a}{\lambda \sqrt{2 \pi r \lambda}} e^{-i r \lambda} \cos (|t| \lambda x) O\left(\frac{1}{\lambda}\right) d \lambda\right| \\
& \leq\left|\int_{C}^{\infty} \frac{\sqrt{2}(\lambda+a)}{\lambda \sqrt{\pi r \lambda}} \cos (r \lambda) \cos (|t| \lambda x) d \lambda\right|+\left|\int_{C}^{\infty} \frac{\sqrt{2}(\lambda+a)}{\lambda \sqrt{\pi r \lambda}} \sin (r \lambda) \cos (|t| \lambda x) d \lambda\right| \\
& +\int_{C}^{\infty} \frac{C_{1} d \lambda}{2 \lambda^{3 / 2}} \\
& \leq|\underbrace{\int_{C}^{\infty} \frac{\lambda+a}{\lambda \sqrt{2 \pi r \lambda}} \cos (\lambda(r+|t| x)) d \lambda}_{\mathcal{I}_{1}}|+|\underbrace{\int_{C}^{\infty} \frac{\lambda+a}{\lambda \sqrt{2 \pi r \lambda}} \cos (\lambda(r-|t| x)) d \lambda}_{\mathcal{I}_{2}}| \\
& +|\underbrace{\int_{C}^{\infty} \frac{\lambda+a}{\lambda \sqrt{2 \pi r \lambda}} \sin (\lambda(r+|t| x)) d \lambda}_{\mathcal{I}_{3}}|+|\underbrace{\int_{C}^{\infty} \frac{\lambda+a}{\lambda \sqrt{2 \pi r \lambda}} \sin (\lambda(r-|t| x)) d \lambda}_{\mathcal{I}_{4}}|+\frac{C_{1}}{\sqrt{C}} .
\end{aligned}
$$

Then

$$
\left|\int_{-1}^{1} \frac{\cos (|t| a x) I_{1}}{\sqrt{1-x^{2}}} d x\right| \leq \int_{-1}^{1}\left(\left|\mathcal{I}_{1}\right|+\left|\mathcal{I}_{2}\right|+\left|\mathcal{I}_{3}\right|+\left|\mathcal{I}_{4}\right|+\frac{C_{1}}{\sqrt{C}}\right) \frac{d x}{\sqrt{1-x^{2}}}
$$


Now

$$
\int_{-1}^{1} \frac{\left|\mathcal{I}_{1}\right|}{\sqrt{1-x^{2}}} d x=\int_{-1}^{1}\left|\int_{C|r+| t|x|}^{\infty} \frac{z+a(r+|t| x)}{\sqrt{2 \pi r z|r+| t|x|}} \cdot \frac{\cos (z)}{z} d z\right| \frac{d x}{\sqrt{1-x^{2}}} .
$$

We have

$$
\frac{1}{\sqrt{1-x^{2}}}\left|\int_{C|r+| t|x|}^{\infty} \frac{z+a(r+|t| x)}{\sqrt{2 \pi r z|r+| t|x|}} \cdot \frac{\cos (z)}{z} d z\right| \rightarrow 0 \quad \text { as } C \rightarrow+\infty
$$

and

$$
\begin{aligned}
& \frac{1}{\sqrt{1-x^{2}}}\left|\int_{C|r+| t|x|}^{\infty} \frac{z+a(r+|t| x)}{\sqrt{2 \pi r z|r+| t|x|}} \cdot \frac{\cos (z)}{z} d z\right| \\
& \quad \leq \frac{1}{\sqrt{1-x^{2}} \sqrt{2 \pi r z|r+| t|x|}} \cdot \sup _{C \geq 0}\left|\int_{C}^{\infty} \frac{z+a|r+| t|x|}{z} \cdot \frac{\cos (z)}{\sqrt{z}} d z\right| \in L_{1}([-1,1])
\end{aligned}
$$

for all $|t| \neq r$ and $x \in(-1,1)$. By the Lebesgue dominated convergence theorem,

$$
\int_{-1}^{1} \frac{\left|\mathcal{I}_{1}\right|}{\sqrt{1-x^{2}}} d x \rightarrow 0 \quad \text { as } C \rightarrow \infty
$$

The proof of

$$
\int_{-1}^{1} \frac{\left|\mathcal{I}_{k}\right|}{\sqrt{1-x^{2}}} d x \rightarrow 0 \quad \text { as } C \rightarrow \infty
$$

for $k=2,3,4$ is similar. Thus the second relation in (29) holds. Running Mathematica 5.0, we get expression (25) for the function $g_{2, r, a}(|t|)$.

\section{The MAIN REPRESENTATiON}

Theorem 8.1. If $n$ is an odd number, then

$$
g_{n, r, a}(|t|)=\frac{g_{1, r, a}(|t|)}{r^{n-1}}+\sum_{k=1}^{\frac{n-1}{2}} \frac{G_{2 k-1}(r, a,|t|)}{r^{n-2 k-1}}+\sum_{k=1}^{\frac{n-1}{2}} \frac{h_{2 k-1, a}(|t|)}{r^{n-2 k+1}},
$$

where $g_{1, r, a}(|t|)$ is defined in Lemma 6.1 .

If $n$ is an even number, then

$$
g_{n, r, a}(|t|)=\frac{g_{2, r, a}(|t|)}{r^{n-2}}+\sum_{k=2}^{n / 2} \frac{G_{2 k-2}(r, a,|t|)}{r^{n-2 k}}+\sum_{k=2}^{n / 2} \frac{h_{2 k-2, a}(|t|)}{r^{n-2 k+2}},
$$

where $g_{2, r, a}(|t|)$ is defined in Lemma 7.1 .

The functions $h_{k, a}(|t|)$ and $G_{k}(r, a,|t|)$ are given by equalities (21) and (22), respectively.

Remark. In contrast to representation (11) of Theorem 8.1 the function $g_{n, r, a}(|t|)$ is expressed in terms of absolutely convergent integrals.

\section{Properties of Weight functions}

We study some properties of weight functions $g_{n, r, a}(|t|)$.

Theorem 9.1. If $n \geq 1$, then

$$
\lim _{|t| \rightarrow+\infty} g_{n, r, a}(|t|)=0 .
$$


Proof. Representation (23) obtained in Lemma 6.1 implies that

$$
\left|g_{1, r, a}(|t|)\right| \leq \frac{1}{\pi r}\left|\log \left(\frac{|t|-r}{|t|+r}\right)\right| \rightarrow 0, \quad|t| \rightarrow+\infty,
$$

for $|t|>r$. The function $g_{2, r, a}(|t|)$ defined by equality (26) can be rewritten as a sum of two integrals, namely

$$
\begin{aligned}
g_{2, r, a}(|t|)= & \int_{0}^{1} \frac{\lambda+a}{r \lambda} J_{0}(|t|(\lambda+a)) J_{1}(r \lambda) d \lambda \\
& +\int_{1}^{\infty} \frac{\lambda+a}{r \lambda} J_{0}(|t|(\lambda+a)) J_{1}(r \lambda) d \lambda .
\end{aligned}
$$

Consider the first integral in equality (30). The representation

$$
J_{\nu}(z)=\frac{(z / 2)^{\nu}}{\Gamma\left(\nu+\frac{1}{2}\right) \Gamma\left(\frac{1}{2}\right)} \int_{0}^{\pi} \cos (z \cos \theta) \sin ^{2 \nu} \theta d \theta
$$

(see [7, §3.1]) implies that

$$
\left|\frac{J_{\nu}(z)}{z^{\nu}}\right| \leq \frac{\sqrt{\pi}}{2^{\nu} \Gamma\left(\nu+\frac{1}{2}\right)}
$$

Thus

$$
\left|\frac{J_{1}(r \lambda)}{r \lambda}\right| \leq 1, \quad\left|J_{0}(|t|(\lambda+a))\right| \leq 1
$$

by inequality (31) for all $\lambda \in[0,1], r>0$, and $|t| \geq 0$. Now we use the asymptotic expansion (2) and obtain

$$
J_{0}(|t|(\lambda+a)) \rightarrow 0, \quad|t| \rightarrow \infty
$$

for all $\lambda \in[0,1]$. By the Lebesgue dominated convergence theorem,

$$
\left|\int_{0}^{1} \frac{\lambda+a}{r \lambda} J_{0}(|t|(\lambda+a)) J_{1}(r \lambda) d \lambda\right| \rightarrow 0, \quad|t| \rightarrow \infty
$$

Consider the second integral in representation (301). Using the bound obtained in Theorem 4.1 of the paper [6], we show that

$$
\left|J_{\nu}(x)-\sqrt{\frac{2}{\pi x}} \cos \left(x-\frac{\pi \nu}{2}-\frac{\pi}{4}\right)\right| \leq \frac{d_{\nu}}{x^{3 / 2}},
$$

where $\nu \geq-1 / 2, x>0$, and $d_{\nu}$ are some constants. 
Applying inequality (32) to the second integral on the right-hand side of equality (30), we get

$$
\begin{aligned}
\left|\int_{1}^{\infty} \frac{\lambda+a}{r \lambda} J_{0}(|t|(\lambda+a)) J_{1}(r \lambda) d \lambda\right| \\
=\mid \int_{1}^{\infty} \frac{\lambda+a}{r \lambda}\left(\sqrt{\frac{2}{\pi|t|(\lambda+a)}} \cos (|t|(\lambda+a)-\pi / 4)+\varepsilon_{0}(|t|, a, \lambda)\right) \\
\leq \mid \\
+\underbrace{\frac{2}{\pi r \sqrt{r|t|}} \int_{1}^{\infty} \frac{\sqrt{\lambda+a}}{\lambda \sqrt{\lambda}} \cos (|t|(\lambda+a)-\pi / 4) \cos (r \lambda-3 \pi / 4) d \lambda \mid}_{\mathcal{J}_{1}} \cos (r \lambda-3 \pi / 4)+\varepsilon_{1}(r, \lambda)) d \lambda \mid \\
+|\underbrace{\frac{\sqrt{2}}{r \sqrt{\pi|t|}} \int_{1}^{\infty} \frac{\sqrt{\lambda+a}}{\lambda} \cos (|t|(\lambda+a)-\pi / 4) \varepsilon_{1}(r, \lambda) d \lambda \mid}_{\mathcal{J}_{2}}| \\
+|\underbrace{\frac{\sqrt{\sqrt{2}}}{\sqrt{\pi} r^{3 / 2}} \int_{1}^{\infty} \frac{\lambda+a}{\int_{1}^{3 / 2}} \cos (r \lambda-3 \pi / 4) \varepsilon_{0}(|t|, a, \lambda) d \lambda}_{\mathcal{J}_{3}}|
\end{aligned}
$$

where

$$
\left|\varepsilon_{0}(|t|, a, \lambda)\right| \leq \frac{d_{0}}{(|t|(\lambda+a))^{3 / 2}}, \quad\left|\varepsilon_{1}(r, \lambda)\right| \leq \frac{d_{1}}{(r \lambda)^{3 / 2}} .
$$

Now we study the asymptotic behavior of the integrals $\mathcal{J}_{i}, i=1, \ldots, 4$, as $|t| \rightarrow \infty$. It is clear that

$$
\begin{aligned}
& \left|\mathcal{J}_{1}\right|=\mid \frac{1}{\pi r \sqrt{r|t|}} \int_{1}^{\infty} \frac{\sqrt{\lambda+a}}{\lambda \sqrt{\lambda}} \cos (|t|(\lambda+a)+r \lambda-\pi) d \lambda \\
& +\frac{1}{\pi r \sqrt{r|t|}} \int_{1}^{\infty} \frac{\sqrt{\lambda+a}}{\lambda \sqrt{\lambda}} \cos (|t|(\lambda+a)-r \lambda+\pi / 2) d \lambda \mid \\
& \leq|\underbrace{\frac{1}{\pi r \sqrt{r|t|}} \int_{1}^{\infty} \frac{\sqrt{\lambda+a}}{\lambda \sqrt{\lambda}} \cos (|t|(\lambda+a)+r \lambda) d \lambda}_{\mathcal{K}_{1}}| \\
& +|\underbrace{\frac{1}{\pi r \sqrt{r|t|}} \int_{1}^{\infty} \frac{\sqrt{\lambda+a}}{\lambda \sqrt{\lambda}} \sin (|t|(\lambda+a)-r \lambda) d \lambda}_{\mathcal{K}_{2}}| .
\end{aligned}
$$

The integrand in $\mathcal{K}_{1}$ changes its sign and assumes zero values at the points

$$
\lambda=\frac{\pi / 2+\pi k-|t| a}{|t|+r}, \quad k \in \mathbf{N} \cup 0 .
$$


Since the lower limit of the integral $\mathcal{K}_{1}$ equals 1 , we derive from (33) that $k \geq s$, where

$$
s:=\frac{r+|t|(1+a)-\pi / 2}{\pi} .
$$

Then

$$
\begin{aligned}
\left|\int_{1}^{\infty} \frac{\sqrt{\lambda+a}}{\lambda \sqrt{\lambda}} \cos (|t|(\lambda+a)+r \lambda) d \lambda\right| \\
=\mid \int_{1}^{\frac{3 \pi / 2+\pi[s]-|t| a}{|t|+r}} \frac{\sqrt{\lambda+a}}{\lambda \sqrt{\lambda}} \cos (|t|(\lambda+a)+r \lambda) d \lambda \\
\quad+\sum_{k \geq[s]+1} \int_{\frac{\pi / 2+\pi k-|t| a}{|t|+r}}^{\frac{\pi / 2+\pi(k+1)-|t| a}{|t|+r}} \frac{\sqrt{\lambda+a}}{\lambda \sqrt{\lambda}} \cos (|t|(\lambda+a)+r \lambda) d \lambda \mid .
\end{aligned}
$$

The series in representation (34) is sign alternating and the absolute value of every term is less than or equal to the absolute value of the preceding term, since $\sqrt{\lambda+a} /(\lambda \sqrt{\lambda})$ is a decreasing function of $\lambda$ for $\lambda>1$. By Leibniz' theorem

$$
\begin{gathered}
\left|\sum_{k \geq[s]+1} \int_{\frac{\pi / 2+\pi k-|t| a}{t \mid+r}}^{\frac{\pi / 2+\pi(k+1)-|t| a}{|t|+r}} \frac{\sqrt{\lambda+a}}{\lambda \sqrt{\lambda}} \cos (|t|(\lambda+a)+r \lambda) d \lambda\right| \\
\leq\left|\int_{\frac{3 \pi / 2+\pi[s]-|t| a}{|t|+r}}^{\frac{5 \pi / 2+\pi[s]-|t| a}{|t|+r}} \frac{\sqrt{\lambda+a}}{\lambda \sqrt{\lambda}} \cos (|t|(\lambda+a)+r \lambda) d \lambda\right| .
\end{gathered}
$$

We derive from (34) and (35) that

$$
\begin{aligned}
\left|\mathcal{K}_{1}\right| & \leq \frac{1}{\pi r \sqrt{r|t|}} \int_{1}^{\frac{5 \pi / 2+\pi[s]-|t| a}{|t|+r}} \frac{\sqrt{\lambda+a}}{\lambda \sqrt{\lambda}} d \lambda \\
& \leq \frac{1}{\pi r \sqrt{r|t|}} \int_{1}^{1+\frac{2 \pi}{|t|+r}} \frac{\sqrt{\lambda+a}}{\lambda \sqrt{\lambda}} d \lambda \rightarrow 0, \quad|t| \rightarrow \infty .
\end{aligned}
$$

A similar reasoning shows that $\left|\mathcal{K}_{2}\right| \rightarrow 0$ as $|t| \rightarrow \infty$. Therefore $\left|\mathcal{J}_{1}\right| \rightarrow 0$ as $|t| \rightarrow \infty$.

Estimating the second integral $\mathcal{J}_{2}$ we prove that

$$
\left|\mathcal{J}_{2}\right| \leq \frac{\sqrt{2} d_{1}}{r^{2} \sqrt{\pi r|t|}} \int_{1}^{\infty} \frac{\sqrt{\lambda+a}}{\lambda^{5 / 2}} d \lambda \rightarrow 0, \quad|t| \rightarrow \infty,
$$

since the integral $\int_{1}^{\infty} \sqrt{\lambda+a} / \lambda^{5 / 2} d \lambda$ converges. Similarly we show that

$$
\left|\mathcal{J}_{i}\right| \rightarrow 0, \quad|t| \rightarrow \infty,
$$

$i=3,4$. Thus $\left|g_{2, r, a}(|t|)\right| \rightarrow 0$ as $|t| \rightarrow \infty$.

It is clear that

$$
\lim _{|t| \rightarrow+\infty}\left|h_{n, a}(|t|)\right|=\frac{a^{n}}{2^{n / 2} \Gamma\left(\frac{n}{2}+1\right)} \lim _{|t| \rightarrow+\infty} \frac{\left|J_{n / 2}(|t| a)\right|}{(|t| a)^{n / 2}}=0 .
$$

Now we show that

$$
\left|G_{n}(r, a,|t|)\right|=\frac{a}{|t|^{n}}|\int_{0}^{\infty} \underbrace{(|t|(\lambda+a))^{n / 2} J_{n / 2}(|t|(\lambda+a)) \frac{J_{n / 2+1}(r \lambda)}{(r \lambda)^{n / 2+1}}}_{S(n, r, a,|t|)} d \lambda| \rightarrow 0
$$


as $|t| \rightarrow+\infty$. To check relation (36) we use the Lebesgue dominated convergence theorem again. In view of the asymptotic behavior of the Bessel function at infinity and at zero,

$$
\begin{gathered}
\frac{a}{|t|^{n}}|S(n, r, a,|t|)| \leq \frac{C}{|t|^{n / 2}} \frac{\left|J_{n / 2+1}(r \lambda)\right|}{(r \lambda)^{n / 2+1}}(\lambda+a)^{n / 2} \in L_{1}([0,+\infty], \\
\frac{a}{|t|^{n / 2}}\left|\frac{J_{n / 2+1}(r \lambda)}{(r \lambda)^{n / 2+1}}(\lambda+a)^{n / 2} J_{n / 2}(|t|(\lambda+a))\right| \rightarrow 0
\end{gathered}
$$

as $|t| \rightarrow+\infty$. Therefore relation (36) holds and the proof of Theorem 9.1 is complete.

Theorem 9.2. If $n \geq 1$, then

$$
\lim _{r \rightarrow \infty} g_{n, r, a}(|t|)=0 .
$$

Proof. Representation (24) of Lemma 6.1 implies that

$$
\left|g_{1, r, a}(|t|)\right|=\frac{1}{\pi r}\left|\pi \cos (a|t|)+\log \left(\frac{r-|t|}{r+|t|}\right) \sin (a|t|)\right| \rightarrow 0
$$

as $r \rightarrow \infty$.

By (3) and [9] we know that

$$
\begin{gathered}
J_{0}(a|t|)=\frac{2}{\pi} \int_{0}^{1} \frac{\cos (a|t| x)}{\sqrt{1-x^{2}}} d x i a|t| J_{1}(a|t|)=\frac{2}{\pi} \int_{0}^{1} \frac{a|t| x}{\sqrt{1-x^{2}}} \sin (a|t| x) d x, \\
\left|g_{2, r, a}(|t|)\right|=\frac{2}{\pi r^{2}} \mid \int_{0}^{1}(\underbrace{\left(1+a \sqrt{r^{2}-|t|^{2} x^{2}}\right) \cos (a|t| x)}_{R_{1}(|t|, r, a, x)} \\
-\underbrace{|t| x\left(a+\frac{1}{\sqrt{r^{2}-|t|^{2} x^{2}}}\right) \sin (a|t| x)}_{R_{2}(|t|, r, a, x)}) \frac{d x}{\sqrt{1-x^{2}}} \mid \rightarrow 0
\end{gathered}
$$

as $r \rightarrow \infty$. Indeed,

$$
\begin{aligned}
& \frac{1}{r^{2}}\left|\frac{R_{1}(|t|, r, a, x)-R_{2}(|t|, r, a, x)}{\sqrt{1-x^{2}}}\right| \\
& \quad \leq \frac{1}{\sqrt{1-x^{2}}}\left|\frac{1+a \sqrt{r^{2}-|t|^{2} x^{2}}}{r^{2}}\right| \cdot\left|1+\frac{|t| x}{\sqrt{r^{2}-|t|^{2} x^{2}}}\right| \in L_{1}([0,1])
\end{aligned}
$$

and

$$
\frac{1}{r^{2}}\left|\frac{R_{1}(|t|, r, a, x)-R_{2}(|t|, r, a, x)}{\sqrt{1-x^{2}}}\right| \rightarrow 0 \quad \text { as } r \rightarrow \infty .
$$

This proves relation (38) by the Lebesgue dominated convergence theorem. Similarly to the proof of (36) (but for the case of $r \rightarrow \infty$ ) we apply the Lebesgue dominated convergence theorem and obtain

$$
\begin{gathered}
\frac{a}{|t|^{n}}|S(n, r, a,|t|)| \leq \frac{C}{|t|^{n / 2}}(\lambda+a)^{n / 2} \mid J_{n / 2}\left(|t|(\lambda+a) \mid \in L_{1}\left(\left[0, C_{1}\right],\right.\right. \\
\frac{a}{|t|^{n}}|S(n, r, a,|t|)| \leq \frac{C}{|t|^{n / 2}} \frac{(\lambda+a)^{n / 2}}{\lambda^{n / 2+1}} \mid J_{n / 2}\left(|t|(\lambda+a) \mid \in L_{1}\left(\left[C_{1},+\infty\right]\right),\right.
\end{gathered}
$$

whence

$$
|S(n, r, a,|t|)| \rightarrow 0 \quad \text { as } r \rightarrow+\infty .
$$

Hence

$$
\left|G_{n}(r, a,|t|)\right| \rightarrow \infty \quad \text { as } r \rightarrow \infty
$$




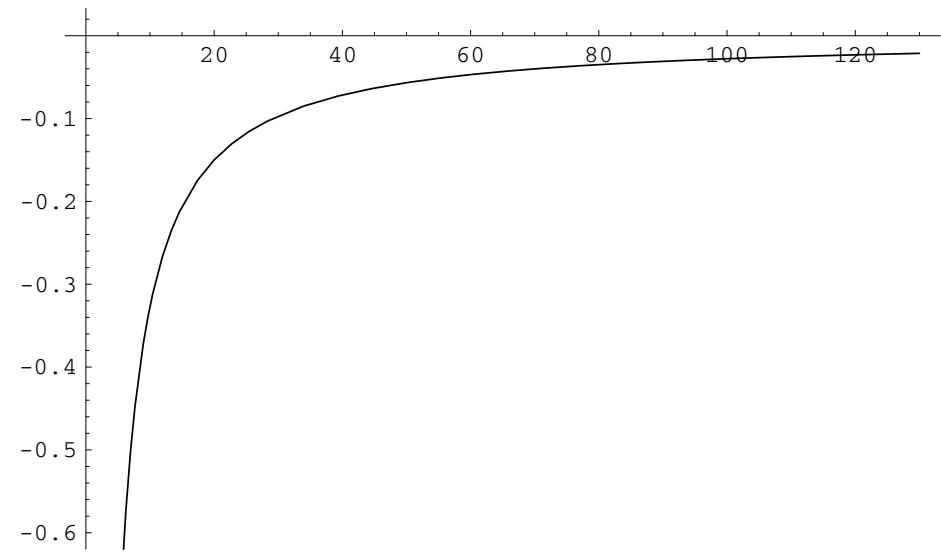

FiguRE 1. $g_{3, r, 1.2}(1)$

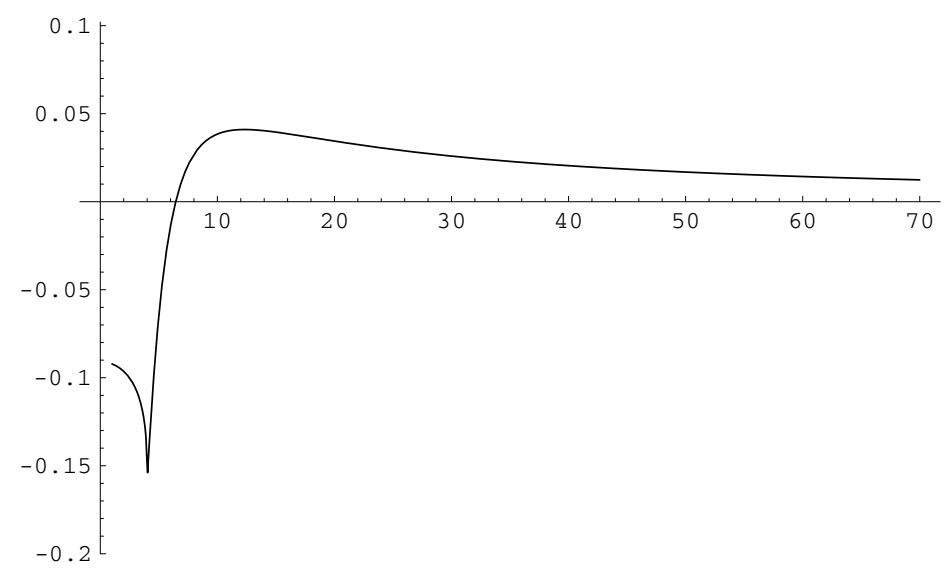

FiguRE 2. $g_{3, r, 1.2}(5)$

Every term in the representation of $g_{n, r, a}(|t|)$ considered in Theorem 8.1 approaches zero as $r \rightarrow \infty$. The theorem is proved.

Example 1. Let $n=3, a=10,|t|=1$, and $|t|=4$. Then the result of Theorem 9.2 is shown in Figures 1 and 2.

Theorem 9.3. The weight function $g_{n, r, a}(|t|), n \geq 1$, is discontinuous if $|t|=r$. This is a discontinuity of the first kind if ar $/ \pi \in \mathbf{N}$; otherwise the discontinuity is of the second kind. 
Proof. The continuity of $G_{n}(r, a,|t|)$ as a function of $|t|$ follows from representation (22) and bound (37). The functions $g_{1, r, a}(|t|)$ and $g_{2, r, a}(|t|)$ are continuous for $|t| \neq r$. Consider the behavior of these functions for $|t|=r$. Lemmas 6.1 and 7.1 imply that

$$
\begin{aligned}
\lim _{|t| \rightarrow r+} g_{1, r, a}(|t|) & =\frac{1}{\pi r} \lim _{|t| \rightarrow r+} \sin (a|t|) \log \left(\frac{|t|-r}{|t|+r}\right) \\
& = \begin{cases}0, & \frac{a r}{\pi} \in \mathbf{N}, \\
-\infty, & \frac{a r}{\pi} \in(2 k, 2 k+1), k \in \mathbf{Z}_{+}, \\
+\infty, & \frac{a r}{\pi} \in(2 k+1,2 k+2), k \in \mathbf{Z}_{+},\end{cases} \\
\lim _{|t| \rightarrow r-} g_{1, r, a}(|t|)= & \frac{1}{\pi r} \lim _{|t| \rightarrow r-}\left(\pi \cos (a|t|)+\log \left(\frac{r-|t|}{r+|t|}\right) \sin (a|t|)\right) \\
= & \begin{cases}\frac{(-1)^{a r / \pi}}{r}, & \frac{a r}{\pi} \in \mathbf{N}, \\
-\infty, & \frac{a r}{\pi} \in(2 k, 2 k+1), k \in \mathbf{Z}_{+}, \\
+\infty, & \frac{a r}{\pi} \in(2 k+1,2 k+2), k \in \mathbf{Z}_{+} .\end{cases}
\end{aligned}
$$

Running Mathematica 5.0 and using [9] we obtain

$$
\begin{aligned}
\lim _{|t| \rightarrow r-} g_{2, r, a}(|t|)= & \frac{J_{0}(a r)-a r J_{1}(a r)}{r^{2}} \\
& +\frac{2}{\pi r^{2}} \lim _{|t| \rightarrow r-} \int_{0}^{1} \frac{a\left(r^{2}-|t|^{2} x^{2}\right) \cos (a|t| x)-t x \sin (a|t| x)}{\sqrt{1-x^{2}} \sqrt{r^{2}-|t|^{2} x^{2}}} d x \\
= & \frac{J_{0}(a r)-a r J_{1}(a r)}{r^{2}}+\frac{2}{\pi r^{2}} \sin (a r) \\
& + \begin{cases}\frac{(-1)^{a r / \pi} \operatorname{Si}(2 a r \pi)}{\pi r^{2}}, & \frac{a r}{\pi} \in \mathbf{N}, \\
-\infty, & \frac{a r}{\pi} \in(2 k, 2 k+1), k \in \mathbf{Z}_{+}, \\
+\infty, & \frac{a r}{\pi} \in(2 k+1,2 k+2), k \in \mathbf{Z}_{+},\end{cases}
\end{aligned}
$$

where $\operatorname{Si}(\cdot)$ is the integral sinus; see 2 .

Furthermore,

$$
\begin{aligned}
\lim _{|t| \rightarrow r+} g_{2, r, a}(|t|)= & \frac{J_{0}(a r)-a r J_{1}(a r)}{r^{2}} \\
& +\frac{2}{\pi r^{2}} \lim _{|t| \rightarrow r+} \int_{0}^{r /|t|} \frac{a\left(r^{2}-|t|^{2} x^{2}\right) \cos (a|t| x)-|t| x \sin (a|t| x)}{\sqrt{1-x^{2}} \sqrt{|t|^{2}-r^{2} x^{2}}} d x \\
& +\frac{2}{\pi r^{2}} \lim _{|t| \rightarrow r+} \int_{r /|t|}^{1} \frac{a\left(|t|^{2} x^{2}-r^{2}\right) \sin (a|t| x)-|t| x \cos (a|t| x)}{\sqrt{1-x^{2}} \sqrt{|t|^{2} x^{2}-r^{2}}} d x \\
= & \frac{J_{0}(a r)-a r J_{1}(a r)}{r^{2}}+\frac{2}{\pi r^{2}} \sin (a r)+\frac{\cos (a r)}{r^{2}} \\
& + \begin{cases}\frac{(-1)^{a r / \pi} \operatorname{Si}(2 a r \pi)}{\pi r^{2}}, & \frac{a r}{\pi} \in \mathbf{N}, \\
-\infty, & \frac{a r}{\pi} \in(2 k, 2 k+1), k \in \mathbf{Z}_{+}, \\
+\infty, & \frac{a r}{\pi} \in(2 k+1,2 k+2), k \in \mathbf{Z}_{+} .\end{cases}
\end{aligned}
$$

These calculations allow one to determine the kind of discontinuity of the function $g_{n}(r, a,|t|)$ for $|t|=r$. This completes the proof of the theorem.

Example 2. Let $n=3, r=1, a=2 \pi$ and $a=5$. The result of Theorem 9.3 is shown in Figures 3 and 4. 


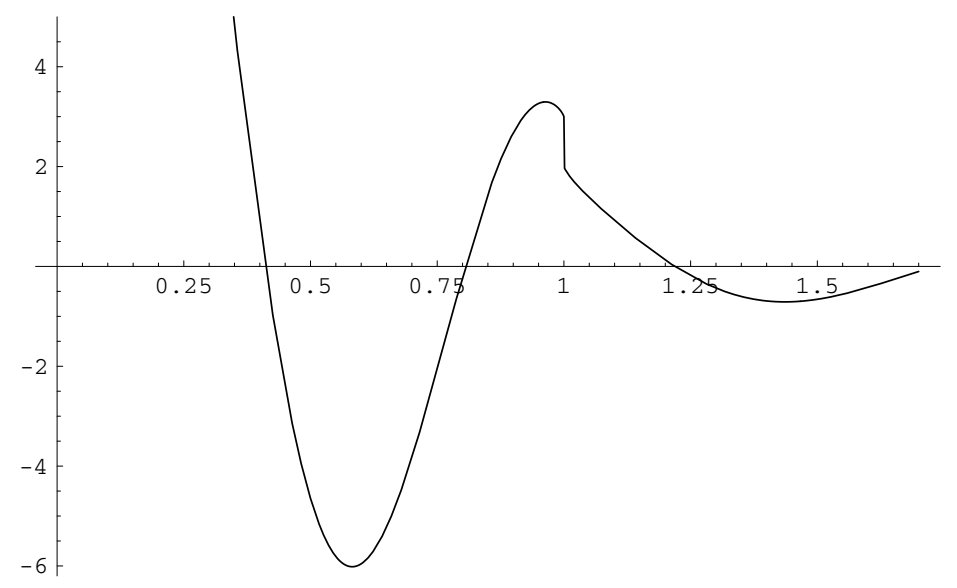

Figure $3 . g_{3,1,2 \pi}(|t|)$

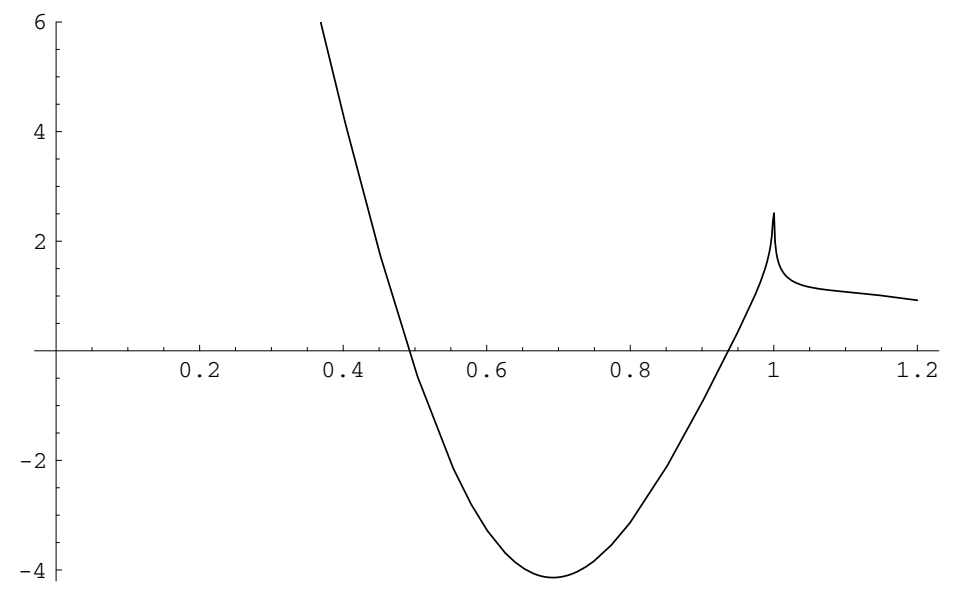

FIGURE $4 . g_{3,1,5}(|t|)$

\section{Concluding Remarks}

We obtained the rate of convergence of function series in the representation of weight functions in Tauberian theorems. We constructed the recurrence relations for weight functions. We studied the asymptotic behavior of weight functions.

\section{BIBLIOGRAPHY}

1. A. Ya. Olenko, A Tauberian theorem for fields with the OR spectrum. II, Teor. İmovirr. Mat. Stat. 74 (2006), 81-97; English transl. in Theory Probab. Math. Statist. 74 (2007), 93-111. MR.2336781(2008i:60085)

2. A. Ya. Olenko, Some properties of weight functions in Tauberian theorems. II, Theory Stoch. Process. 13(29) (2007), no. 1-2, 194-204. MR2343823 
3. N. N. Leonenko and A. V. Ivanov, Statistical Analysis of Random Fields, "Vyshcha Shkola", Kiev, 1986; English transl., Kluwer Academic Publishers Group, Dordrecht, 1989. MR917486 (89e:62125)

4. M. I. Yadrenko, Spectral Theory of Random Fields, "Vyshcha Shkola", Kiev, 1980; English transl., Optimization Software, Inc., Publications Division, New York, 1983. MR.590889 (82e:60001)

5. A. Ya. Olenko and B. M. Klykavka, Some properties of weight functions in Tauberian theorems I, Theory Stoch. Process. 12(28) (2006), no. 3-4, 123-136. MR2316570

6. A. Ya. Olenko, Upper bound on $\sqrt{x} J_{\nu}(x)$ and its application, Integral Transforms Spec. Funct. 17 (2006), no. 6, 455-467. MR2238583 (2008c:33003)

7. G. N. Watson, A Treatise on the Theory of Bessel Functions, Cambridge University Press, Cambridge, 1995. MR1349110 (96i:33010)

8. A. Erdelyi, W. Magnus, F. Oberhettinger, and F. Tricomi, Higher Transcendental Functions, vol. 1, Mc.Graw-Hill, New York, Toronto, London, 1953. MR.0058756 (15:419i)

9. A. Erdelyi, W. Magnus, F. Oberhettinger, and F. Tricomi, Tables of Integral Transforms, vol. 1, McGraw-Hill, New York, Toronto, London, 1954. MR0061695 (15:868a)

Department of Probability Theory and Mathematical Statistics, Faculty for Mechanics and Mathematics, National Taras Shevchenko University, Academician Glushkov Avenue 6 , KYIV 03127, UKRAINE

E-mail address: bklykavka@yahoo.com

Received 25/DEC/2006

Translated by S. KVASKO 